\title{
MAŁGORZATA GRYGIELEWICZ
}

(Paryż, Université Paris VIII)

\section{LA RENCONTRE PHILOSOPHIQUE DANS LE JARDIN GREC}

«En Grèce il n'y avait pas de jardin » est une assertion bien connue. On la trouve dans la majorité des histoires de jardins écrites par différents spécialistes. Cela vient du fait que l'on associe les Grecs à la naissance de l'agora, lieu central de la ville où les citoyens se réunissent pour parler. Elle est employée communément par opposition aux jardins « orientaux» et 《romains» qui évidemment dominent dans les sources. Pourtant Homère donne une description d'un jardin des Phéaciens et de Laërte, la mythologie parle de jardin des Hespérides, et d'Adonis nous connaissons le jardin d'Epicure...

Toutes les civilisations ont élaboré et développé des formes différentes de jardins selon leurs préoccupations. Tous ces jardins, créés dans des cités du monde entier à des époques et dans des conditions très contrastées, reflètent de façon fidèle l'image de l'esprit de la société. Esprit qui exprime le sentiment et la pensée sur Dieu, sur le monde et sur la culture. Le jardin est toujours l'expression de la mémoire, de la tradition et de l'engagement pour le futur. Le jardin est un élément d'architecture et l'architecture est toujours un reflet de l'ordre qui règne à un moment précis. Cet ordre a sa résonance dans la mentalité des gens. Par sa coexistence avec le temps et sa situation à la fois dans l'architecture de la ville et dans la nature paysagée, il jette une passerelle entre elles, dessinant et interrompant leurs limites. Nous devons parler du jardin grec comme d'un phénomène, bien entendu différent du jardin romain, du jardin moderne mais présent dès l'Antiquité grecque. Ce qui est caractéristique du jardin, c'est qu'il se situe au début de la formation de la «culture». Les recherches, et ensuite la découverte de sa signification sont le résultat de la séparation entre la dimension culturelle et la dimension naturelle. La dimension culturelle en se distinguant de celle de la 
nature, ne s'en sépare pourtant pas. Le rôle du jardin souligne cette séparation, qui, en même temps, conjugue deux sphères opposées. Pour survivre dans un environnement hostile l'homme se trouve obligé de créer le jardin. Il est nécessaire de récolter pour vivre mais aussi de vivre pour récolter et le jardin est indispensable à cet acte. Certains peuvent associer les jardins au concept de clôture. Le jardin comme un lieu séparé, exclu du reste, ordinairement clos. Le mot jardin exprime la fascination qu'exercent les choses secrètes et la promesse d'ouverture que suggère l'inconnu. La simple masse des matériaux, des sources épigraphiques et archéologiques a quelquefois menacé d'éclipser la question de base. Au cours des dernières années, la discussion critique des principes et des concepts a progressé autour de la philosophie antique, éclairant un problème central dans l'étude de l'Antiquité et dans l'histoire du genre humain. Néanmoins il y a encore, dans l'étude de ce qu'on appelle les histoires de jardin grec, des clichés et des préjugés qui doivent êtres récusés, puisqu'ils conduisent à des demi-vérités, voire à une absolue méprise.

Demandons-nous au départ : qu'est ce que le Jardin ? Si on pose cette question et si nous nous proposons de produire un discours pour y répondre, d'emblée celui-ci s'effondre à peine l'avons-nous entamé. Nous nous rendons compte que nous ne savons plus trop que dire, l'objet même qui était en question paraît se dérober. C'est à cette situation bizarre que nous allons devoir nous affronter, au double phénomène de jardin philologique et philosophique qui prend son origine à l'époque antique grecque. Il nous faut souligner que, premièrement, le jardin n'est pas un objet au sens usuel que le mot possède dans le dictionnaire et l'encyclopédie - étant absent dans les dictionnaires de la philosophie, et deuxièmement le langage est mis en échec devant l'exigence d'avoir à en parler. Nous sommes souvent contraints de parler de ce dont nous ne savons pas comment parler. Néanmoins nous allons demander à être guidés dans les jardins, les forêts et les champs d'écriture en souhaitant comprendre son énigme, énigme philologique et philosophique à la fois. L'histoire d'un objet est toujours liée le plus étroitement à la représentation que nous nous en faisons. Conformément à cette représentation il est déjà déterminé, ce qu'on considère comme important et intentionnel à ce sujet. Nous rappelons que le jardin est lié au concept de clôture, que le jardin c'est l'expression de la mémoire, un reflet de l'ordre qui règne, qui se place au début de la formation de la «culture», qui jette une passerelle entre la 《 nature » et la « culture », qui est la séparation et en même temps conjugue deux sphères opposées, sachant neanmois que le mot «culture » est un mot d'orygine latin et n'a pas d'equivalent semantique en grec ancien. Le problème c'est de savoir comment se mettre à traiter un sujet, dont le nom est bien connu, quand on sait approximativement ce qu'il est ? La méthode de travail nous demande d'abord de nous éloigner de la définition commune de ce sujet. Après avoir atteint une 
distance suffisante, essayer de décrire différemment, en utilisant les « mots» et les « choses » qui viennent des champs éloignés de notre sujet. Afin d'en dégager la charge historique, celle qui inéluctablement nous cache l'essence du sujet, il faut que nous oubliions l'histoire du jardin pour parvenir à son origine ! Nous allons seulement mentionner l'importance de la réception dans les recherches sur le jardin, car pour raisonner les problèmes de critique du texte il est nécessaire de disposer du savoir non seulement sur les «mots» mais aussi sur les « choses ». Le jardin n'était jamais considéré comme un simple jardin, mais comme le témoignage de quelque chose d'autre, comme le siège de la nature apprivoisée. Le langage grec exprime la contradiction selon laquelle une chose est à la fois elle-même et autre chose identique et non identique. L'un est le tout, tout est dans l'un.

L'environnement habité par les dieux, des lieux de naissances divines et des mariages sacrés nous amènent à la typologie du jardin: jardins des dieux, jardins des citoyens, jardins des philosophes, jardins poétiques, jardins d'amour, jardins d'écriture, jardins funèbres... Tous ces jardins viennent d'une conception de la vie, d'un ancrage dans la vie. En visitant tous ces jardins, nous devions nous pencher sur leurs racines, pour y trouver leur sens exact et pas seulement une proliférations des idées enfermées dans un cadre quelconque de muraille. Pour ces recherches, il a fallu revenir à l'origine du jardin. Avant la pratique de quelque chose il en faut trouver son idée. L'idée de jardin a commencé avec Homère, à cette époque il avait son idée précise et sa notion précise, voir le vocabulaire. Bien entendu les jardins de l'époque homérique n'étaient pas semblables aux réalisations tardives qui s'approchaient des réalisations contemporaines. Néanmoins nous y trouvons une situation flagrante, les Grecs de l'époque homérique ont inventé une forme de jardin, ils ont influencé d'autres formes de jardins.

Pour comprendre la signification du jardin et son rôle chez les Grecs, il était nécessaire de parvenir à son origine. Si la notion du jardin doit être établie non de façon arbitraire, mais philologique, alors un commentaire de ce genre devient une «philologie ». On pourra admettre qu'à l'égard du jardin, la seule possibilité de procéder, c'est de rechercher et de comparer ce qui était nommé jardin dans la littérature grecque. Nous rappelons que les textes que nous possédons ne représentent guère qu'un fragment parcellaire d'écriture antique. Une grande question qui surgit à chaque moment du travail est : quel est le rapport de la partie sauvée à la masse des œuvres qui a été perdue ? A cette question a essayé de répondre «la critique du texte antique»qui poursuit les traces de l'œuvre jusqu'à nous, pour restituer sa forme la plus authentique, c'est-à-dire établie par son auteur. La critique du texte est à côté de l'interprétation un des principaux aspects de la philologie antique. La tentation de parvenir au texte original, après avoir éliminé plusieurs différentes versions, c'est le devoir du philologue. Souvent sa volonté « d'objectiver» l'œuvre l'éloigne de la «vie»du texte, qui est arrivé 
jusqu'à nous grâce à sa 《réception » toujours nouée dans les préjugés idéologiques du passé. C'est dans cette «vie» que nous avons voulu replacer le jardin grec. Replacer et non déplacer!

Nous souhaitons nous projeter dans l'espace du jardin et explorer des no-

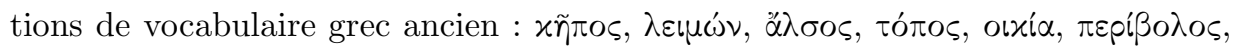

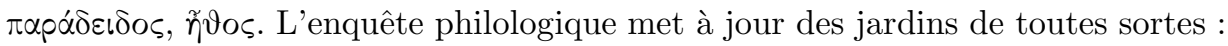
ceux de la géographie mythique comme : le jardin d'amour, le jardin paradisiaque et utopique, le jardin homérique, et ceux de la géographie réelle, comme les jardins d'Athènes, associés à Platon, Aristote, Epicure et d'autres philosophes. A la tête des investigations se place le mot $\chi \tilde{n} \pi$ s qui sert de cadre pour l'école d'Epicure. Tous ces termes de jardin dont nous parlons sont si polysémiques qu'ils évoquent plusieurs formes de jardin et n'exposent pas un type de jardin précis et distinctif. Tous les jardins dont nous parlons : jardin de profit, jardin fleuri, jardin d'Adonis, jardin arboré, les vignes, jardin d'agora, jardin sacré de bosquet et jardin de cimetière, montrent une large catégorisation du jardin par les Grecs. Toutefois cette subdivision du jardin est trop détaillée et paraît mélanger les critères de différenciation, et surtout, elle perd son aspect métaphorique, si présent encore dans le jardin d'Adonis ou des Hespérides par exemple.

Nous commencons nos investigations dans la géographie mythique de la Grèce, nous traversons les jardins d'amour pour nous arrêter face aux arbres afin d'en cueillir les fameuses pommes des Hespérides. Poursuivant Eros nous traversons les prairies et les bois sacrés pour enfin entrer dans les jardins fleuris et sentir les roses, les lys, et cette plante énigmatique connue sous le nom de Moly. Le récit du jardin d'Eden croise le mythe d'Eurydice qui, avant de mourir, blessée par un serpent, cueillit des roses dans un jardin. On pourra chercher une analogie entre le désir de la connaissance absolue venant de la Bible et la tradition grecque du récit sur Orphée qui est allé à Hadès chercher sa femme. Elle était une dryade, nymphe de la forêt qui incarnait l'arbre. La descente aux enfers d'Orphée à la recherche de sa femme et de la connaissance absolue, qu'on ne peut pas atteindre sur la terre et que finalement on ne peut pas voir avec « les yeux vivants » est vraisemblable. Orphée nous apparait d'un côté comme un « faible» jardinier, perdu dans le brouillard des malheurs, du pessimisme et de la faiblesse, il annonçait l'école d'Epicure. D'un autre côté, le chanteur divin se manifeste comme un brave héros des Argonautes. Il nous accompagna dans nos recherches d'une pensée au fond invisible, cachée à Hadès qui disparaît aussitôt sortie dans le monde de l'apparence. Même si la pensée est invisible, elle signifie la nécessité de recul au monde et c'est le jardin qui permet son apparence. Le jardin, qui possède sa propre luminosité, nous guérit par la lumière comme Orphée guérit par la sienne, Orphée (du phénicien: «Aour» la lumière et «Rophae» guérison, celui qui guérit par la lumière). Nous poursuivrons notre périple, guidés par deux personnages, par 
Ulysse, qui à la fin de son voyage a retrouvé son jardin paternel : «Ulysse se perd pour se retrouver ; il s'éloigne de la nature en s'abandonnant à celle-ci, qu'il devra affronter dans chaque aventure et, comble de l'ironie, cette nature impitoyable triomphera lorsque ce héros impitoyable rentrera au pays, devenu autant que juge et vengeur, l'héritier des puissances auxquelles il a échappé ${ }^{1}$ et par Orphée qui n'était pas heureux comme Ulysse et qui n'a pas «fait un beau voyage...» Peut-être est-il arrivé en retard à la « rencontre» dans le jardin philosophique ? Emportés par les différents courants d'eau, nous nous sommes infiltrés dans les versets d'Homère et les jardins de Calypso, d'Alcinoos et de Laërte. Quittant les temps fabuleux nous avons décrypté le jardin dans la géographie réelle de Hellade. Nous nous sommes promenés dans les jardins funéraires et nous avons visité les premiers parcs, dont : l'Académie de Platon, le jardin d'Aristote, de Théophraste pour arriver au jardin d'Epicure. A la fin nous nous sommes approchés des animaux qui habitent dans les jardins, pour conclure que l'homme étant un animal, il était un jardin. Nos flâneries de jardin dans l'espace philologique s'achevèrent dans le jardin d'Adonis, un véritable jardin d'écriture, où des lettres et des pensées formèrent l'unité de tout.

Dans l'approche qui nous intéresse particulièrement, celui qui prend pour base la création du jardin et son utilisation nous allons aborder le problème de son fonctionnement dans son contexte socio-culturel. Comme le disait Pline l'Ancien : « Maintenant il nous reste à revenir à la culture des jardins, qui se recommande et par elle-même, et par ce que l'Antiquité a admiré avant tous les jardins des Hespérides, ceux des rois Adonis et Alcinoüs. $»^{2}$

Les Grecs étaient un peuple à l'instinct grégaire, amoureux des villes, avec un esprit communautaire développé. Ils adoraient les interminables discussions, c'était un peuple pour lequel la vie publique comptait plus que la vie privée. Ils discutaient dans les rues et sur la place de l'agora et ils ont fini par s'intéresser aux jardins qui servaient de cadre à ces discussions, puis ont cherché à les développer. Mes recherches autour du jardin ne se concentrent pas sur l'aspect anthropologique de la vie en Grèce, même si la confrontation avec le territoire géographique pourra m'aider à assimiler la pensée grecque sur le jardin. L'intérêt de me déplacer en Grèce naît du réel besoin de rencontre avec « la terre », « la région» et « le

1 Theodor W. Adorno et Max Horkheimer, La dialectique de la raison, Gallimard, Paris, 2004, p. 62 .

2 Pline L'Ancien, Histoire Naturelle, En collaboration avec Agnès Vinas, (site Méditerranées), traduction, E. Littré, Paris, 1848-1850; livre XIX. 19, IV.

Naturalis Historia, Plinius Secundus, selon : the Thesaurus Linguae Graecae, the Packard Humanities Institute, The Perseus Project and others. Dans Diogenes version 3.1.6,

19.49.1. « $\mathrm{Ab}$ his superest reverti ad hortorum curam et suapte natura memorandam et quoniam antiquitas nihil prius mirata est, quam Hesperidum hortos ac regum Adonidis et Alcinoi itemquepensiles $»$. 
séjour » grecs où je peux pleinement assister au déploiement de l'intuition philosophique qui en est issue. Comme disait Heidegger lors de son voyage en Grèce : « Toutes choses auxquelles il nous est difficile de réfléchir tant que continue à nous échapper ce qui a défini le monde grec dans ce qu'il a de plus propre. Et la question se pose encore de plus belle : où devons-nous rechercher ce qu'il a de plus propre ? $»^{3} \mathrm{Il}$ est vrai que toute visite en tout endroit où le jardin a habité, œuvré et assisté aux fêtes nous plonge dans une perplexité toujours croissante. Toutefois avant d'y entrer, il faut se reposer autour de midi dans l'herbe haute sous de vieux arbres dans les parages d'Attique et chercher des papillons voletant et rendant le silence plus perceptible, pour saisir un très léger pressentiment du moment où Pan a son heure ${ }^{4}$. Nous y sommes attirés par la promesse de quelque chose de grec. « Les doutes persistaient sur le point de savoir si une expérience de ce qui est initialement grec nous serait jamais encore accordée ; si, comme c'est bien connu, toute expérience de cette sorte n'était pas déterminée d'avance par l'horizon chaque fois actuel de celui qui l'éprouve et bornée en conséquence; des doutes quant à savoir si, de ce fait, tout effort tenté pour regarder le commencement n'était pas vain et inefficace, même s'il venait à réussir à l'intérieur de certaines limites ; des doutes quant à savoir si le simple fait de penser à cela ne gâtait pas la croisière dans son expérience immédiate. ${ }^{5}$ Pourtant la Grèce a été assez souvent décrite de façon pertinente à maints égards et avec compétence, mais les jardins, nous ne les voyons pas souvent dans ces investigations, pourtant ils sont partout devant nous.

Pour les Grecs, amoureux des cités, la terre n'avait pas beaucoup d'importance. Pline le Jeune parle pour la première fois des jardins publics de Grèce, ou des jardins semi-publics des philosophes grecs. Grâce à lui, nous savons que sur les hippodromes toscans il y avait plus de verdure qu'il n'y en eut jamais sur un gymnase grec, et, plus remarquable encore, que les statues ont disparu. Sans statue et sans urne, l'atmosphère de bosquet sacré grec n'existe plus. Plotin, qui encourage à sculpter sa propre statue, reflète bien le caractère de la Grèce antique dans ces mots : «Retourne en toi-même et vois. Et si tu ne vois pas encore ta propre beauté, fais comme le fabricant qui doit rendre une statue belle : il enlève ceci, efface cela, polit et nettoie jusqu'à ce qu'une belle apparence se dégage de la statue ; de même pour toi, enlève le superflu, redresse ce qui est tordu et, purifiant

3 M. Heidegger, Séjour, Aufenthalte, Edition bilingue, traduction, postface et notes de François Vezin, Editions du Rocher, Paris, 1992, p. 37.

4 C'est une heure où le soleil culmine dans le ciel et brûle la terre. Les oiseaux cessent de chanter, les serpents se cachent sous les pierres et les insectes font silence. Les Anciens, qui pressentaient les mystères de la nature, connaissaient bien le sens de cette heure et l'appelaient « l'heure de Pan ».

5 M. Heidegger, Séjour, Aufenthalte, p. 21. 
tout ce qui est ténébreux, travaille à être resplendissant. Ne cesse à sculpter ta propre statue, jusqu'à ce que brille en toi la splendeur divine de la vertu et que tu vois la tempérance qui siège sur son « auguste trône ». ${ }^{6}$ Pierre Hadot commente les mots de Plotin : «Une expression - sculpter sa propre statue - est d'ailleurs souvent mal comprise, car on s'imagine facilement que [...] elle signifierait : prendre une pose, choisir une attitude, se composer un personnage. En fait il n'en est rien. Pour les Anciens Grecs en effet, la sculpture était un art qui «enlève» par opposition à la peinture qui est un art qui «ajoute»: la statue préexiste dans le bloc de marbre et il suffit d'enlever le superflu pour la faire apparaître. ${ }^{7}$ Comme le disait Plotin il faut devenir le regard même, sans aucun obstacle à son unification, et comme une lumière véritable, non dimensionnée, une lumière absolument sans mesure, pénétrée à l'intérieur. Il faut devenir une vision, confiante en soi, fixer son regard et voir en soi-même. Nous trouvons chez les Romains à la place de la statue, une statuaire d'art topiaire, c'est-à-dire l'art de « sculpter » les arbres absolument inconnu chez les Grecs. La quête de la réalisation de soi a été remplacée par un esthétisme moral. Pline l'Ancien écrit : i Tel est le platane nain, qui doit ce nom à sa petitesse forcée; car nous avons trouvé l'art de faire avorter même des arbres. Ainsi donc, parmi les végétaux aussi, il sera question de la disgrâce des nains. Ce résultat s'obtient et par la manière de planter et par celle de tailler. C. Martius, de l'ordre équestre, ami du dieu Auguste, inventa, il y a moins de quatre-vingts ans, l'art de tondre les bosquets. $\rangle^{8}$

Dans la littérature grecque on trouve trois approches typiques de représentations du jardin. Ainsi Homère valorisait la nature sauvage, Hésiode souli-

6 Plotin, Ennéades, Traduit par Jérôme Laurent, Sur lebeau, I, 6 [9], dans Plotin, Sur le beau et autres traités, trad. sur la dir de Luc Brisson et Jean-François Pradeau, Flammarion, 2008. "A

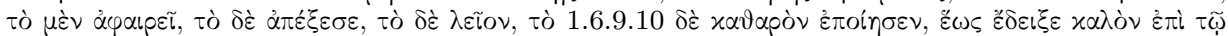

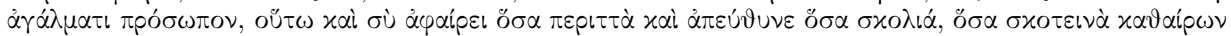

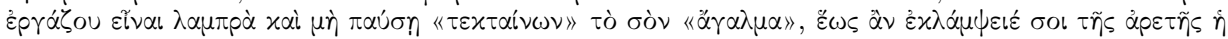

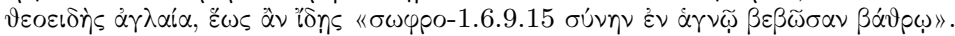

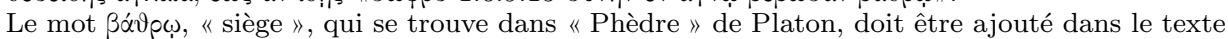
de Plotin, pour que la phrase offre un sens. Voir : Plato Phil., Phaedrus, éd. Burnet, J. Oxford:

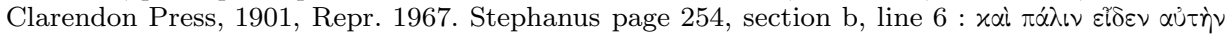

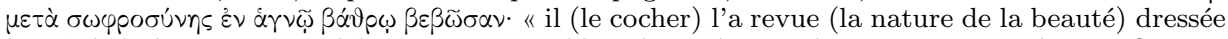
à coôté de la sagesse et debout sur son piédestal sacré. » trad. Luc Brisson, Platon, Oeuvres complètes. Flammarion, 2008.

7 Pierre Hadot, Exercices spirituels et la philosophie antique, Albin Michel, 2002, p. 62.

8 Pline L'Ancien, Histoire Naturelle, traitant des Arbres. Texte français Paris : Dubochet, 1848-1850. édition d'Émile Littré, Livre Naturalis Historia XII, VI.

Naturalis Historia, Plinius Secundus, selon : the Thesaurus Linguae Graecae, the Packard Humanities Institute, The Perseus Project and others. Dans Diogenes version 3.1.6,

12.13.1 Namque et chamaeplatani vocantur coactae brevitatis, quoniam arborum etiam abortus invenimus. Hoc quoque ergo in genere pumilionum infelicitas dicta erit. fit autem et serendi genere et recidendi. primus C. Matius 12.13.5 ex equestri ordine, Divi Augusti amicus, invenit nemora tonsilia intra hos LXXX annos. 
gnait l'aspect pragmatique de cultiver la terre et Anacréon présentait son aspect érotique. Nous souhaitons démontrer que les premiers témoignages des jardins grecs sont toujours liés au contexte religieux et que le jardin en Grèce antique est lié aux deux sphères opposées, celle de la nature et celle de la culture. L'intention de démarcation est évidente car si chaque forme de culture humaine était jardin, le jardin comme tel n'existerait pas. Dans une vision plus générale, ce que l'on peut prendre comme le fondement de la singularité du jardin est son appartenance, car tous les jardins dépendent des dieux ou des gens. A l'époque, quand les dieux habitaient chaque élément de la terre et donc de la nature, quand ils étaient présents à chaque instant de la vie humaine, quand il n'y avait pas de limites nettes entre ce qui est sacré et profane, seuls existaient les jardins divins. Les Grecs, pour s'approprier des lieux de la nature, construisaient des temples dédicacés aux dieux particuliers. Ils étaient entourés par un enclos servant de résidence sacrée appelée

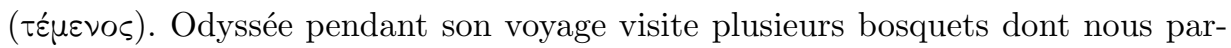
lons. Bien souvent ce ne sont pas des descriptions détaillées ; Homère, dans ses fragments, ne mentionne que les endroits. Durant le périple d'Odyssée, c'est le chemin qui devient le plus important, il se déroule et scande les points d'arrêt. Et quand Odyssée, après mille ruses arrive en héros au but de son voyage, dans la ville natale d'Ithaque tant espérée, il dit :

« Je le comprends, j'y songe et j'avais prévu ton invite.

Partons donc, et sers-moi de guide jusqu'au bout!

mais, si tu en as de coupé, donne moi un bâton

Pour m'appuyer, puisuque vous dites le chemin glissant."

Il dit, jeta sur son épaule une affreuse besace

toute trouée; la bretelle était une corde;

puis Eumée lui donna le bâton qu'il lui demanait.

Ils partirent tous deux, les chiens et les bouviers, la bas, gardaient la ferme, il menait en ville son maitre

sous l'aspect d'un vieillard et d'un pitoyable méndiant appuyé sur sa canne, habillé de tristes haillons.

Mais lorsque descendu le chemin caillouteux,

Ils approcherèrent de la ville et atteignirent la fontaine

en pierre aux belles eaux où puisent les gens de la ville,

Fontaines due à Ithacos, Nérite, Polyctor

- un bois de peupliers nourris par l'eau montait

en cercle tout autour, et l'eau froide coulait

du sommet d'une roche; au-dessus se dressait l'autel

dédié au Nymphes où tout passant dépose son offrande. ${ }^{9}$

9 Homère, Odyssée, trad. Philippe Jaccottet, livre XVII, 193-213, Homeri Odyssea, éd. von 
Ces enclos existaient partout sur le territoire de la Grèce : Un temple d'Aphrodite et de Héphaïstos à Athènes, le temple de Posejdon à Istmos, d'Asclepios à Epidauros, d'Appolon près de Sparte ou de Dionisos à Brazyaj. Nous les trouvons aussi sur la côte asiatique comme le temple d'Aphrodite en Syrie. Pindar, dans une de ses Odes nous rappelle : «Mais tandis que les accents de la gloire retentissent dans les jardins délicieux de Cyrène, dans ces bosquets consacrés à Vénus, n'oublie pas de rapporter à la divinité, comme à son premier auteur, la félicité dont tu jouis. » ${ }^{10}$ Ces résidences sacrées, qui formaient le cadre de l'éducation grecque - nommé $\left\langle x \alpha \lambda\right.$ ox $\alpha \gamma \vartheta^{i} \alpha{ }^{11}{ }^{11}$, avec le temps se transformaient en lieux de rencontres philosophiques et faisaient office de jardins publics. Leur caractère sacré était connu et lisible pour les Grecs aussi bien à l'époque homérique qu'à la fin de la période hellénistique.

Les villes grecques étaient petites, il n'y avait pas de grande possibilité pour créer de fastueux jardins privés. Néanmoins nous y trouvons les premiers parcs publics avec leurs promenades plantées d'arbres, leurs sièges et leurs terrains de jeux. Les arbres monstrueux, plantés loin de la ville à la campagne sauvage, symbolisent la nature qui doit s'effacer devant une civilisation à venir. D'autres arbres, comparés aux hommes, qui, comme les feuilles mortes avec le vent s'épandent tour à tour sur le sol des forêts verdoyantes puis dans la terre fertile, dans la nouvelle vie symbolisaient la pérennité humaine. Les arbres embrassent tout l'environnement

der Mühll, P., Helbing \& Lichtenhahn, Basel, 1962.

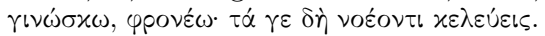

$\alpha \lambda \lambda^{\prime}$ '̆

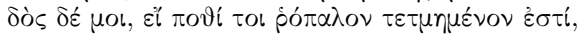

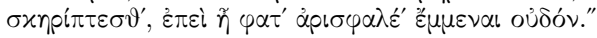

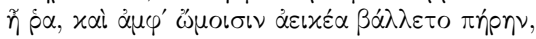

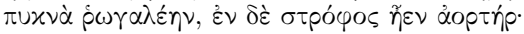

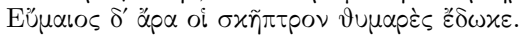

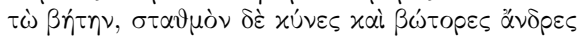

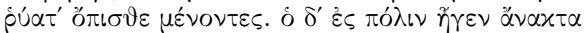

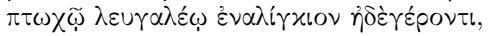

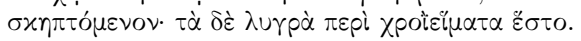

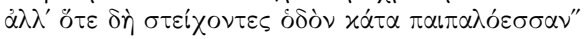

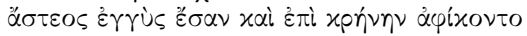

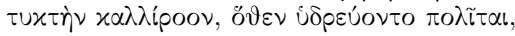

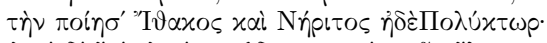

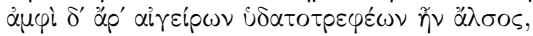

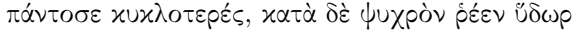

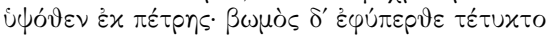

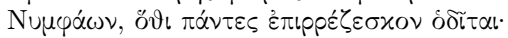

10 Pindar, Odes - Les Pythiques, ode V:

$\tau \widetilde{\omega} \sigma \varepsilon \mu \grave{\eta} \lambda \alpha \vartheta \varepsilon \dot{\varepsilon} \tau \omega$,

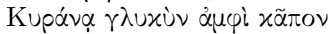

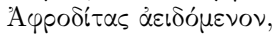

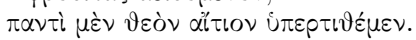

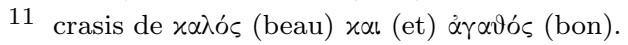


grec (à l'exception des bocages en grec $\alpha^{\prime} \lambda \sigma o \varsigma$ ), habité par les dieux et les hom-

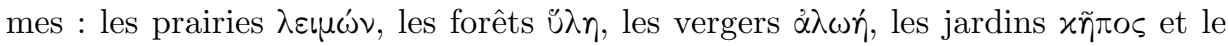
paradis $\pi \alpha \rho \alpha ́ \delta \varepsilon\llcorner\delta o \varsigma$. La forêt U் $\lambda \eta$, qui est habitée par le dieu Pan se transforme de sa forme totale et universelle en forme locale, susceptible de s'adapter à différents usages des hommes en jardin. Xénophon écrivait que «le paradis » avant qu'il ne devienne une plantation divine était un lieu planté d'arbres. Ces lieux paradisiaques, comme le jardin des Hespérides et le jardin d'Eden avec les arbres de la connaissance et de la vie sont toujours les lieux sacrés et archaïques qui gardent la mémoire. L'origine sacrée n'a jamais disparu, même si seule a subsisté l'ambiance créée par les vestiges purement matériels. Parlons de jardin stérile d'Adonis, de jardin d'écriture. Comme disait Platon l'écriture ne produit nul fruit, nulle vérité, parce qu'elle est comme le jardin d'Adonis : stérile et périssable. Écrire, c'est comme cultiver les jardins d'Adonis, passer son temps à s'amuser. Il s'agit de la question du divertissement lié à la beauté du jardin, qui commence à se définir comme un endroit de repos et non de récolte. Le jardin d'Adonis, qui est le jardin d'écriture n'est pas un jardin philosophique. En dernier lieu, nous avons identifié le jardin philosophique comme la préfiguration du jardin d'Epicure. Le philosophe n'y est pas encore le jardinier, il se compare néanmoins à un jardinier, non de la terre fertile mais de l'esprit, et il ne cultive pas des plantes mais des pensées. Il évoque le travail de la terre, le labeur et la patience qui sont les techniques de plantation et d'entretien. Après Platon c'est Aristote et surtout Théophraste qui promulguèrent la posture du scientifique qui collecte, inventorie et rédige les sommes de la connaissance botanique. Avec Epicure le jardin d'esprit deviendra le jardin de réalité. Et tout cela à travers l'image du jardin éphémère d'Adonis.

\section{L'EAU ELEMENT DE LA SYNTHESE}

L'eau, un des éléments qui constitue le monde et la vie, une matière sacralisée par des manifestations divines qui est en même temps une matière indispensable à la vie, une matière à la fois profane et sacrée. Nous distinguos trois jardins différents associés aux trois sources d'eau, le jardin de la mer, le jardin terrestre et le jardin de la rivière. La stérilité de l'eau salée est équilibrée par sa fonction transitoire. L'eau circule dans la terre. L'eau, ce sont aussi les nuages, qui habitent deux jardins situés aux deux confins opposés. L'un de ces jardins est le jardin de leur père Océan, et l'autre est leur mère la Terre. La préoccupation majeure des jardiniers était l'arrosage. Pour cultiver il faut arroser et ici l'eau douce est très précieuse car elle n'est jamais abondante. Mais nous aussi remarquons qu'Esope dit que de toute façon, les plantes sauvages poussent mieux que celles, cultivées 
dans un jardin par un jardinier. Pour lui le jardin paraît comme une maison d'orphelinat pour des enfants, comparés aux plantes, abandonnées par leur mère. Les sources d'eau répondent aux veines dans lesquels coule le sang. Platon et Aristote nous fourni une réflexion intéressante, que la création de l'homme répond à la création du jardin, obligé d'être hydraté.

Le génie de la «synthèse grecque »se reflétait dans toutes ces investigations. Dans la culture grecque, toutes les actions humaines créaient une totalité unifiée. Le jardin faisait partie de la nature et de la culture. Ses démarcations et contradictions se définissent progressivement. Même à l'époque hellénistique, les œuvres de la littérature grecque portent les signes d'une unité originale de la nature et de la culture, d'ordre éthique et esthétique. La présence du jardin dans la littérature témoigne de l'origine religieuse de la littérature et donc de l'art. C'est en effet la religion qui a formé son cadre originel. La recherche de l'unité de l'homme, qui ne connaissait pas l'aliénation, se manifeste dans la création et la redécouverte du jardin comme symbole d'une harmonie primordiale, qui irrémédiablement passait. Le retour intuitif à l'unité de l'homme, s'est accompagné d'un rapprochement avec la nature et il est toujours lié au désir de changement. Ce changement de perspective montre le désir qui règne dans la société, le désir de révolte ou de réforme radicale intérieure du présent régime social. Ce désir se reflète dans le retour du poème idyllique significatif des périodes mouvementées de l'histoire. Nous retrouvons dans la littérature romaine cette tendance à traiter la structuration du jardin comme lutte contre la désintégration intérieure de l'homme. Dans la tradition grecque elle apparaît progressivement pour se manifester clairement à l'époque hellénistique. La question de la recherche de chemins potentiels dans la littérature grecque, inspirés par l'image du jardin comme expression d'affectation à une nouvelle synthèse est une hypothèse. Nous l'héritons des Romains, pour qui le jardin était déjà un concept bien délimité et plus uniforme que pour les Grecs. Pline l'Ancien écrit : «Aujourd'hui on possède dans Rome même, sous le nom de jardins, des lieux de plaisance, des campagnes, des villas. L'usage en a commencé à Athènes par Épicure, maître en fait de vie oisive: jusqu'à lui on n'avait pas su habiter la campagne à la ville. ${ }^{12}$ Un médiéviste russe, D. S. Lichaczov écrit : « la disparition des capacités de «lecture» des jardins comme d'un système psychologique et de capacité de réception de leur «climat esthétique» d'époque peut être expliqué par le fait que pendant ces cent dernières années la capacité de perception iconographique et la connaissance générale des symboles et des emblèmes

12 Pline l'Ancien, Histoire Naturelle, éd. d'Émile Littré, Naturalis Historia, Plinius Secundus, selon : the Thesaurus Linguae Graecae, the Packard Humanities Institute, The Perseus Project and others. Dans Diogenes version 3.1.6, éditzeur inconnu, livre, 19, 1V. iam quidem hortorum nomine in ipsa 19.51.1 urbe delicias agros villasque possident. primus hoc instituit Athenis Epicurus otii magister; usque ad eum moris non fuerat in oppidis habitari rura. 
traditionnels se sont estompées de façon drastique. ${ }^{13}$ Nous n'extrapolons pas ici sur les raisons de cette ignorance, mais une raison que l'on peut aisément indiquer est celle de la disparition de l'éducation classique et théologique. La perception iconographique des systèmes de jardins est particulièrement difficile aussi parce que dans les jardins, plus souvent que dans les autres œuvres apparaît une symbolique cachée, les schémas iconologiques cachés. Lichaczow qui explore le rôle du jardin dans la culture occidentale comme un espace culturel et idéologique, compare le jardin à une «bibliothèque spéciale». La capacité de déchiffrement de son espace culturel décide de notre conscience culturelle. La bibliothèque est un endroit où sont réunis et rangés des notes, des écrits, des livres. L'idée de la bibliothèque existe depuis l'apparition de l'écriture. Avant, les mots et les phrases étaient ordonnés dans la mémoire des hommes, qui eux-mêmes savaient bien retrouver le fragment et le récit voulus, comme les horticulteurs qui connaissaient les plantes de leur jardin. Le mot bibliothèque était précédé par un autre mot, qui correspondait à une classification des paroles d'artistes, un recueil de morceaux

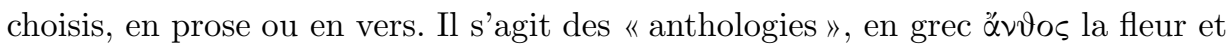
$\lambda \varepsilon^{\prime} \gamma \omega$ qui après le sens premier, qui signifie « dire-parler» se traduit par cueillir, trier. Il est synonyme de «florilège ». Les anthologies nous renvoient directement au jardin pour ramasser les fleurs et composer des bouquets. Pour les sentir et les reconnaître il fallait connaître leurs noms. Les roses qui poussent dans les jardins d'amour, des lys qui son nées du lait divin de la déesse Héra, les crocus qui forment sa couche avec Zeus, les jacinthes qui nous introduisent dans les prairies humides. Du jardin d'Adonis, nous connaissons les anémones qui poussent des gouttes de son sang qui empourprèrent la terre. Elles symbolisent la mort et le renouveau de la nature. Une autre fleur après le crocus, nous introduit à l'Enfer, c'est asphodèle, il fleurit la tombe des morts. L'iris unit la terre au ciel portant une écharpe aux sept couleurs de l'arc-en-ciel... Dans le livre « Bouquet d'Athéna » ${ }^{4}$ Helmut Baumann associe les noms des plantes aux couleurs, parfums, textures. La nature riche des sensations surgit des mots riches d'émotions. D.S. Lichaczow nous avertit que depuis la Renaissance nous perdons cette capacité de lecture. A quoi bon tenter de déchiffrer le jardin grec, dont nous avons peu de vestiges tangibles et ceux qui nous permettent seulement de présenter le problème? Peut être pour l'opposer à la grande présence du jardin hébraïque qui a tant influencé notre imaginaire occidental?

13 Cité d'après, Obraz parku - ogrodu a alternatywa ewolucji literackiej, w: Przestrzen ogrodu, przestrzen kultury, Universitas, Krakow, 2008, p. 101, traduction personnelle.

14 Baumann Helmutt, Le Bouquet d'Athéna les plantes dans la Mythologie et art grecs, Maison Rustique, 2008. 


\section{RENCONTRE DEHORS DES JARDINS D'HISTOIRE}

Nous constaterons que le jardin est au cœur de la « rencontre ». La rencontre pour nous la plus remarquable est celle des Grecs et des Hébreux, mais la rencontre des traditions, des religions, des philosophies, des mentalités, est bien plus intéressante. Il faut multiplier les rencontres. C'est la question du devenir d'un jardin. Le jardin, qui est au cœur de la «rencontre» et qui y occupe une place privilégiée, à côté de l'architecture, de la sculpture ou de la peinture, se situe hors de la durée, et parfois en défi au temps. Le jardin meurt et renaît à chaque saison ; il est dans une transformation continue, à mi-chemin entre l'éternel et l'instantané. Le jardin ne crée pas un objet, et tout son artifice se borne à rendre sensible la beauté qu'il révèle et réunit en un même lieu. Le jardin n'est guère qu'une façon de prendre possession de la nature. Le jardinier s'empare des arbres, des fleurs, des cascades ; il devient le maître de la nature tandis qu'elle-même est tempérée dans ses caprices, ses excès et son exubérance. Aussi le jardin est d'abord un choix : il immobilise et reproduit à « loisir » ce que la nature ne donne qu'au gré des conditions physiques.

Avant la rencontre il y a la promenade dans les jardins. Une forme de vagabondage disait Jean Toussaint Desanti. Promenade comme acte, comme politique, comme expérimentation, comme vie, - disait Deleuze. Une promenade qui s'engage sur les chemins de la pensée, similaire à une herbe, qui pousse en son milieu, une promenade qui est une promesse de rencontre. Les concepts philosophiques seraient alors comme des herbes de différentes tailles et forces, des herbes qui poussent toujours au milieu, on peut marcher sur elles ou les éviter, pour ne pas les écraser. Ou encore elles seraient comme des sons, des couleurs, des images, des intensités qui nous conviennent ou non, qui passent ou ne passent pas pour redessiner les contours - s'ils existent - du jardin. Et le jardin serait alors un gazon, ou plutôt une herbe de connaissance du bien et du mal, ou encore une herbe de vie? Cette herbe qui pousse au milieu en redessinant le chemin de notre promenade, fait du jardin grec un acte de pensée dont la vie est une puissance non personnelle. Henry Miller disait que «L'herbe n'existe qu'entre les grands espaces non cultivés. Elle comble les vides. Elle pousse entre - parmi - les autres choses. La fleur est belle, le chou est utile, le pavot rend fou. Mais l'herbe est débordement, c'est une leçon de morale. ${ }^{15}$ Notre jardin grec serait alors comme une herbe et nous, ses lecteurs et ses chercheurs comme des bisons qui cherchent ces herbes parmi les autres. L'ensemble de ce travail forme un tout semblable à une pelouse ou un champ

15 Cité dans : Gilles Deleuze, Dialogues, avec Claire Parnet, Ed. Flammarion, Paris, 1977, p. 37. 
d'herbes sauvages et greffées. Les concepts mentionnés se croisent et grandissent tous en même temps. Le développement de l'idée de jardin se déploie progressivement et graduellement du premier au dernier et régulièrement sur l'ensemble des niveaux. Cela permettra d'imaginer le jardin dans son ampleur, du début de sa conception, et d'oublier le caractère évolutif de l'idée de jardin contemporain. Ce travail de jardin s'apparente à la vision libre sur la variabilité de ce que le jardin représente. Ce n'est que sous l'horizon de l'incessante nouveauté du changement qu'apparait la plénitude du détail et des faits.

On peut résumer la Bible dans une phrase comme «l'exode», la sortie, un mouvement perpétuel au-dehors. Tout dépend de ce dehors, surtout la création du monde. La conception de la création du monde, qui est fondamentale pour la théologie biblique est la conception de la création de l'histoire. Les deux se lient dans un rapport logique et ontologique. La création du Monde biblique est un premier moment d'histoire. Au commencement du Monde se situe la conception du jardin qui n'est pas encore fermé car il n'a pas à être fermé. Le jardin se ferme au moment de «la sortie» des premiers hommes. Il se ferme aux péchés des premiers hommes et à leur histoire. Il initie le voyage vers l'avenir, vers un nouveau «topos» qui est pour nous connu comme « utopie». Tout se renverse de façon dialectique, au point que la création n'est plus au commencement mais à la fin de notre chemin. Le besoin d'une nouvelle création apparait. L'eschaton d'une nouvelle terre et d'un nouveau ciel. Une escalade vers la nouvelle Jérusalem, où, ni le soleil ni la lune n'éclairent, aucune lampe divine, seulement le fils de Dieu. L'idée de jardin biblique naît au moment de la naissance de l'histoire, au moment de la sortie du jardin, à ce moment, à côté de ses sources, se placent les anges qui gardent leurs «frontières». Ils gardent la mémoire du jardin, un ingrédient indispensable de l'histoire. De la mémoire qui, par définition, doit être limitée. L'homme qui a quitté le jardin d'Eden, désire dans l'euphorie atteindre «l'horizon», et sans ce désir l'homme n'est pas authentique. L'homme, est un fou fugitif au-dehors. Il est comme Odyssée qui marche vers Ithaque pour après la quitter perpétuellement. L'homme est la négation irréductible de fermeture. Il est une ex-plosion pure. (Deleuze)

La réalité dialectique du jardin décrite par Homère et dans la Bible, définit la culture de l'Occident, qui a résulté de la confrontation dialectique entre le composant (com-poser) grec et le décomposant (de-com-poser) judéo-chrétien. D'après ces deux traditions nous héritons d'une extension de sens abstraite du jardin. Elle est habitée sans conteste possible par ces sortes de jardin d'une habitation au premier abord invisible, que la méditation philosophique sur le jardin ainsi que sa réception, permettent alors de faire entrevoir. Allons à la rencontre du jardin philosophique. 


\section{LES JARDINS ABSTRAITS DE LA PHILOSOPHIE}

Nous voudrions relier la question du jardin à celle de l'abstraction la plus avancée, abstraction radicale. Non pas à la figure abstraite de la mort ou de la vie qui se place au cœur de la question du jardin, mais aux formes de la pensée sur la mort et sur la vie qu'on va relier à tel endroit, l'endroit qui pouvait être le jardin. Pour jouer l'abstraction, peut-être faut-il d'abord se retirer dans un jardin philosophique, voire s'isoler dans un jardin et raconter une histoire longue qui ne soit pas un mythe. L'histoire du jardin n'est aucunement l'histoire du mythe. On ne peut pas dissocier un discours sur le jardin d'un discours sur les mythes grecs, néanmoins il n'y a pas de mythe du jardin. Sortons du jardin homérique, jardin de Calypso, jardin d'Alcinoos et de Laërte, demandons nous : où est le jardin maintenant? Le jardin grec et le jardin d'aujourd'hui?

Supposons qu'il y ait une figure exemplaire et inédite du jardin, voire du jardin radical qui paraisse marquer le temps de notre civilisation. C'est à identifier ce jardin de la rencontre philosophique qu'on accèdera. Allons au déracinement de l'abstraction, en passant par les multiples jardins, par les lieux d'abstraction que sont : le jardin d'amour, paradis et jardin utopique, jardin de la polis grecque, cimetières, et entrons dans le jardin d'écriture, puis changeons la route et tournons dans l'intérieur du jardin, dans le jardin et cyberespace, dans le jardin et espace temps virtuel, puis dans le jardin de la guerre, pour mesurer un traité à ces thèmes, dans l'économie des pages qui nous est assignée. Allons à la rencontre du jardin philosophique.

Pour éclaircir l'énigme du jardin grec nous en appellerons à des philosophes : F. Nietzsche, M. Heidegger, H. Arendt, G. Deleuze, M. Foucault, J. Derrida et L, J.T. Desanti afin d'élaborer un concept du jardin philosophique. Le terme « jardin » conférera à une vocation conceptuelle, confirmera que les notions du vocabulaire philosophique : comme jardin révolutionnaire (F. Nietzsche), Da-

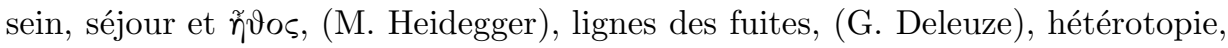
(M. Foucault) et khôra, (J. Derrida) jardin non cultivé, (L. Kolakowski)... sont des étapes sur les chemin de la pensée. Ces étapes nous rapprocheront des formes du jardin philosophique, sans exposer un type de jardin particulier avec des critères précis de distinctions. Après avoir utilisé le mot «concept» il faudrait ajouter que les Grecs nommaient opıøuós le concept lui-même c'est-à-dire limite. Les Grecs ont observé que la limite répas, n'est pas l'endroit où quelque chose s'arrête. Au contraire, à partir de là, quelque chose commence à être. Ce changement de perspective, qui, encore une fois, peut perturber notre manière de penser, nous permet d'avancer dans le concept de jardin, qui parfois nous semble ne pas exister, est présent ensuite, réellement et formellement.

Tout le problème de la philosophie consiste à prendre en charge l'environne- 
ment du monde dans lequel on est, avec ses voisinages, avec ses rapports qui se construisent et qui donnent sens à ce voisinage. Cela nous permet de penser cet environnement, de lui donner une forme. La forme du jardin qu'il nous faut élargir perpétuellement, essayer de comprendre le rapport à un autre monde que ce jardin qui ne cesse jamais d'être là. Et plus nous nous en éloignons, plus le jardin viendra avec nous. La relation réciproque de l'homme et de l'espace est son habitation pensée. Dans cette habitation réside le rapport que l'homme entretient avec les lieux et les espaces. Le jardin, qui est un espace à part, isolé, retranché, un écart demandant une clôture, à mi-chemin des deux « dangers » de la nature et de la société, le jardin d'Epicure est un espace remarquable pour la pensée grecque. Il n'est pas un intermédiaire, il n'est pas la réduction à l'échelle humaine de la Nature. C'est par une séparation d'avec elle qu'il se constitue. Point d'intersection de données souvent antinomiques, à mi-chemin entre nature et culture, vie et mort, tantôt œuvre de Dieu, tantôt celle du démon, le jardin d'Epicure transcende, il est une œuvre de l'homme qui illustre une métaphore de l'être. Nous voudrions relier la question du jardin à celle de l'abstraction radicale. Non pas à la figure abstraite de la mort où de la vie qui se place au cœur de la question du jardin mais aux formes de la pensée sur la mort et sur la vie qu'on va lier

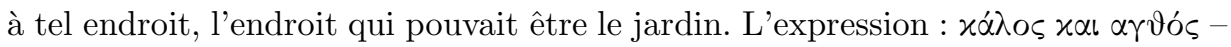
(beau et bon) qui résume bien l'idéal grec de perfection humaine nous servira à démontrer l'évolution du rapport des Grecs avec la nature et son renversement dans l'antagonisme (anti-agonisme). Comme de nombreux hellénistes, à commencer par Nietzsche, l'ont remarqué, que pour un Grec ancien, la laideur était une réfutation, la vérité sur le jardin, celle que nous chercherons, peut être considérée comme une sorte d'apparence. La tradition philosophique a modifié le fondement de l'apparence en sa cause, en lui accordant une réalité supérieure à ce qui est donné aux sens. En considérant que l'opinion sur la supériorité de la cause, qui est au-dessus de l'effet, est la plus vieille et la plus durable erreur métaphysique j'indiquerai la source de cette confusion et le constat que les phénomènes, non seulement découvrent mais aussi cachent quelque chose, ou plutôt, en découvrant quelque chose, ils cachent quelque chose de différent (Héraclite). Hannah Arendt en renversant cette vielle hiérarchie métaphysique, acclame la suprématie de la surface. Et ce n'est pas un simple renversement. En acceptant l'accord de l'Etre et de l'Apparence, elle rejette la dichotomie elle-même de l'Etre vrai et de simple Apparence. Elle souligne la suprématie de ce qui s'apparaît sur ce qui ne s'apparaît pas...

Le philosophe en se mettant à l'écart du monde des phénomènes attendait quelque chose qui apparaîtrait à l'esprit comme une chose apparaît aux yeux, est-il important ce lieu de la manifestation, celle du jardin ?

Rappelons qu'interroger les Grecs c'est paradoxalement apprendre à différer 
d'eux et à inventer ce qui nous est propre, inventer les conditions de l'actuel qui est comme dit Nietzsche inactuel et intempestif. Et bien comme Ulysse, qui est

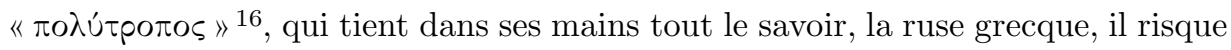
le détour, il se jette sur les côtés, il s'ouvre aux intempéries, aux circonstances, aux événements, il s'offre au donné dur dont il ne fera jamais système pour ne pas le voir s'évanouir dans la norme. Cela implique la pensée en termes d'événement, et non la pensée en référence à la stabilité, tranquillité constante. Penser veut dire prendre le risque de décentrement, le mouvement, le départ, bref comme dit Deleuze - se mettre en route. Ce qui est premier dans la pensée, c'est l'effraction, la violence c'est l'ennemi. ${ }^{17}$ L'important est de se mettre en route, toujours devant un inconnu, de ne pas se trouver sur la terre déjà habitée, l'important est de ne rien « reconnaître ». Accompagnons la catabase d'Ulysse et d'Orphée, les deux personnages hors série, exceptionnels par leur ruse et leurs pouvoirs, capables de commander aux vents ou de ramener un défunt de l'Hadès. Comme l'écrit J. P. Vernant : « chez les hommes divins, si divers qu'ils soient, c'est l'individu humain qui prend l'initiative, mène le jeu et passe de l'autre côté. Grâce aux pouvoirs exceptionnels qu'il a su acquérir, il peut quitter son corps abandonné comme en état de sommeil cataleptique, voyager librement dans l'autre monde et revenir sur terre ayant gardé le souvenir de tout ce qu'il a vu dans l'au-delà. ${ }^{18}$ Revenons alors sur la terre du jardin, là où la philosophie, en transposant dans son propre registre le thème de voyage, nous conduira à la rencontre, là où la pensée ne s'aligne pas avec le conformisme, où elle ne s'aligne avec rien qui implique le jugement de valeur ou de reconnaissance. Au lieu de régler, juger et reconnaître, la pensée de jardin trouve, vole et rencontre. La pensée a pour essentiel la rencontre. La rencontre philosophique dans le jardin grec est une rencontre dehors, pour se trouver dehors. Se déterritorialiser, c'est quitter une habitude, une sédentarité. Plus clairement, c'est d'échapper à une aliénation, à des processus de subjectivation précis ${ }^{19}$. La déterritorialisation qui n'est jamais une fin en soi, est sans retour. Une fois que la conscience trouve son chez soi, son territoire, elle y reste jusqu'à la prochaine déterritorialisation prochaine rencontre. Le jardin de la rencontre philosophique au cœur duquel nous méditons est un jardin de solitude épicurien. Comme disait G. Deleuze : «quand on travaille on est forcément dans une solitude absolue. Il n'y a de travail que noir et clandestin. Seulement c'est

16 Поһútрoтоs - qui se tourne en beaucoup de sens, qui parcourt mille lieux divers, souple, habile, selon dictionnaire grec français de Anatole Bailly, Librrairie Hachette, 1957.

17 G. Deleuze, Différences et répétitions, p. 181.

18 J. P. Vernant, Mythe et religion en Grèce ancienne, édition du Seuil, 1987, p. 111.

19 G. Deleuze, F. Guattari, Félix, Capitalisme et schizophrénie. L'Anti - Edipe, Ed. Minuit, 1972 , p. 162. 
une solitude extrêmement peuplée. Non pas peuplée de rêves, de fantasmes, de projets, mais de rencontres. Une rencontre c'est peut-être la même chose qu'un devenir ou des noces. C'est du fond de cette solitude qu'on peut faire n'importe quelle rencontre. ${ }^{20}$

\section{CONSCIENCE INTIME DU JARDIN}

Les Grecs sont comme des enfants, naïfs - disait Nietzsche, et cruels - disait Heidegger. Il est vrai qu'il faut préserver les enfants de la brutalité du monde mais nous devons aussi préserver le monde de la fraicheur et cruelle innocence des enfants. Toute la philosophie depuis les Grecs contribue à cet acte de préservation, parfois certains philosophes nous rapprochent de l'enfance grecque, les autres, de façon déterminée nous en éloignent. En traversant les pensées des philosophes différents, en sortant des écrits des poètes grecs nous devons nous mettre en quête, si possible, du germe de jardin. Car, en suivant cette voie, nous espérons pouvoir montrer l'investissement réciproque du jardin au sein de la pensée: c'est-à-dire repérer le surgissement de la forme de jardin originaire, qui nous porte à désigner ce quelque chose qui paraît minime dans la plénitude d'une présence au monde. De toute façon l'homme ouvre pour que la Terre devienne sa patrie et pour la rendre habitable. Le grec ne se salit pas les mains et n'investit pas la terre pour qu'elle devienne sa propriété. Il joue sur la terre comme les enfants contemporains jouent dans le « jardin de Jordan ${ }^{21}$. Le grec comme un enfant nomade prend le chemin pour arriver dans la ville. Sur son chemin les enfants ramassent cailloux, branches et avec ces objets construisent les murs de la ville. ${ }^{22}$ La diversité du

20 Gille Deleuze, Dialogues, avec Claire Parnet, Ed. Flammarion, 1977, p. 13.

21 Le nom "Jardin de Jordan" ne vient pas de la Bible mais du nom d'un médecin Henryk Jordan habitant à Cracovie en XIX siècle. Il a inventé et créé le premier jardin des enfants de ce type dans sa ville, il a été inauguré en 1889 sur une surface de 22 hectares. A ce moment Cracovie était sous la domination autrichienne, cela était très important pour l'idée de Jordan, parce que son jardin (parc) de détente et de jeux dédicacé aux enfants était riche de symbolique patriotique polonaise. Après la guerre (14-18) l'idée du jardin de Jordan, qui était trop coûteuse fut remplacée par les aires de jeux, plus petites et sans verdure.

22 Dans diverses activités ludiques l'enfant s'habitue à l'application des règles et des lois sociales. Il familiarise son futur outil de travail. Chez Platon dans sa vision de la société idéale nous trouvons deux termes (paideia et agôn) pour designer les jeux d'enfants et d'adolescents. L'éducation des enfants présentée dans la «République » diffère de celle présentée dans les 《Lois ». («Les Lois» 789) Il s'agit de l'âge de passage de trois ans : dans la République l'enfant est enfermé à la crèche, la cellule familiale est supprimée. Dans les Lois, il reste à l'âge de trois ans dans la famille, et pendant ses premières trois années il est sous la surveillance de sa mère. Il n'est plus enfermé dans la crèche et il peut se déplacer (avant l'obtention de la marche, sur les bras de sa mère) entre la maison familiale (ojkos) et la cité - (polis). Un des lieux de leur jeu est le 
monde qui l'entoure, des objets qu'il rencontre sur son chemin de promenade désignent l'horizon de sa perception. Mais intuitivement il cherche autre chose. La fascination de la ville disparaît et pousse vers la sortie de la ville. Les enfants n'oublient jamais de chercher «le lieu», objet de son désir. Les enfants ont bâti l'agora et s'enfuient dehors, dans une autre hétérotopie, plus ouverte et à la fois hostile, dans la nature arrangée, dans le jardin. Les enfants enchantés (grisés) par leur babillage, concentrés au milieux de la polis ont pris un chemin pour arriver au monde désenchanté du dehors. Regardons de près l'image métaphorique de recherche d'un autre horizon que nous apporte Jeaan-Toussaint Desanti :

Imagine donc des voyageurs qui auraient entrevu de haut et de loin, au-delà d'une forêt, un lieu leur convenant et auraient formé le projet d'y habiter. De là-haut n'apercevraient aucun chemin capable d'y conduire. Parvenant dans la plaine leur situation s'inverserait. La forêt qui les sépare du séjour espéré comporterait un excès de pistes, de sentiers et de routes, qui se recouperaient sans cesse en croisements labyrinthiques. La forêt perverse les égarerait à chaque pas. S'en remettant au hasard, ils finiraient, après bien des essais, par découvrir un chemin de bonne apparence, large et droit. Or ce chemin conduirait à une ville, cachée dans la forêt, invisible des hauteurs, une grande ville pleine de monde, comportant une multitude de places publiques, de monuments, de lieux de spectacle. Ils interrogeraient les habitants. «Où est le chemin qui conduit à ce lieu que nous avons entrevu de là-haut »? Toujours on leur répondrait : «Quel lieu ? Il n'y a pas de lieu, pas de route autre que celle qui, par hasard, vous a conduit jusqu'à ici. Elle est aussi la route du retour. Dans cette ville, c'est par où l'on entre que l'on sort. Au-delà c'est la forêt. Nous pensons qu'elle est infinie, ou tout comme, puisqu'il est impossible d'y avancer, fût-ce d'un seul pas. De toute manière, tout ce que vous pouvez faire dans notre ville c'est de la visiter et d'y demeurer juste le temps d'y apprendre notre histoire et nos façons de vivre. Après, il vous faudra repartir par où vous êtes venus. Ce lieu n'est pas un séjour pour les autres. A vrai dire ce n'est pas non plus un lieu de passage : il n'est comporte pas d'au-delà habitable. »

Sans doute nos voyageurs accepteraient-ils l'invitation. Ils s'instruiraient et s'amuseraient beaucoup dans la ville. Au bout d'un temps, monument, spectacles, bibliothèques n'auraient plus de secret pour eux. Certain même deviendraient des experts en ces matières. Peut-être oublieraient-ils ce lieu entrevu depuis les hauteurs, objet de leur désir ancien.

Un jour, pourtant (telle était la loi de la ville), il leur faudrait s'en aller par le seul chemin possible qui les ramènerait « là-haut ». Parvenus à ce point de départ,

sanctuaire avec ses jardins, ses temples, ses édifices. Voir : Djibril Agne (Dakar), « Identification des objets et obéissance aux règles et aux lois par le jeu. Une étude parallèle chez Platon et dans quelques sociétés traditionnelles ouest - africaines ». L'article dans « Histoire, espaces et marges de l'antiquité : hommages à Monique Clavel-Lévêque, volume 4, Par Marguarite Garrido - Hory, Antonio Gonzalès. p. 223. 
il se mettraient à discuter, en proie à une agitation extrême : « Faut-il en croire nos yeux ? Cette forêt que nous apercevons dans la plaine est touffue, mais petite et nous croyons bien voir d'ici son au-delà et, en lui, le lieu objet de nos désirs. Il lui est impossible, telle que nous le voyons, de dissimuler et si riche de culture et d'histoire. Et pourtant nous avons séjourné dans la ville, parlé à ses habitants, et nous nous y sommes instruits. De quels mirages sommes nous la proie ? ${ }^{23}$

Mais, oublier la ville...Qu'est ce que cela signifie? Quelles sont les conséquences de cet oubli ? Oublier la ville implique un déplacement, c'est un appel à 《oublier» tout ce que les façons de parler ordinaires, irréfléchies, réfléchies, savantes et théoriques, nous livrent concernant l'expérience de notre vie dans la ville en direction du dehors, du jardin. Il faut alors oublier les murs de nos villes et leur place bien établie. Et aussi ce qui sommeille dans l'usage des villes : les institutions, les lois, les certitudes, les séquences légalisées de la sécurité de nos actes. La ville que nous tenons pour la forme du « cours universel des choses » objective nos pensées. Il faut alors de nouveau établir, ce qui nous paraît nécessaire, un mouvement vers le dehors de la ville, pour sortir de l'agora, sortir dans le jardin.

Dans la «Condition de l'homme moderne»H. Arendt ${ }^{24}$ écrit que la terre est la quintessence même de la condition humaine, et que la nature terrestre pourrait bien être la seule de l'univers à procurer aux humains un habitat où ils puissent se mouvoir sans effort et artifice. Terre est ce qui nous est premièrement donnée, elle n'est pas habitable en tant que telle. Mais c'est l'œuvre du jardin qui rend la terre habitable pour créer la passerelle entre la nature et l'architecture en bâtissant un Monde commun. Parce que le Monde doit être construit et transmis, grâce à un jardin placé sur la Terre primordiale. C'est l'œuvre poétique qui a en particulier, par la trace de paroles qu'elle laisse, la propriété de durer. Les jardins d'Adonis, stériles de la pensée et les anthologies, peuplées des différentes fleurs se réunirent sur la Terre, ils nous offrent les possibilités de chemin. D'où vient ce chemin? Et où mène-t-il? Amène t-il au jardin au bout du chemin ou est-ce un chemin qui ne mène nulle part? Une fois il amène assurément là où il serait possible d'avoir une vue des environs tout à fait dégagée qui révèlerait sans effort à l'horizon la complexité du paysage. Une autre fois il s'enfoncera dans les méandres de la pensée close et fermée à toutes les issues, pour sombrer dans l'obscurité. Ce chemin n'allait assurément pas sans détours, retours et égarements. Cette succession de tentatives d'arriver aux différents jardins, inévitablement se soldera par un échec,

23 Jean-Toussaint Desanti, Réflexion sur le temps, Conversation avec Dominique-Antoine Grisoni, Grasset, 1992, p. 95.

24 H. Arendt, Condition de l'homme moderne, traduction française G. Fradier, Calmann-Lévy, 1961, 1983, réédité avec une préface de Paul Ricoeur, Pocket, 1988, 1992 (The Human Condition, London, Chicago, University of Chicago Press, 1958). 
où à la fin on ne saurait toujours pas de façon claire et distinct ce que c'est au juste le «jardin», qui n'est pas censé être clos, qui peut être avec ou sans murailles.

Le jardin c'est une ancienne « destination de l'homme », il n'est pas une nouvelle anthropologie philosophique fondée de manière ontologique (M. Heidegger) ni une anthropologie historique fondée de manière philologique (C. Calame). Sa fondation répond à l'angoisse la plus ancienne, celle de la mort et finitude de la vie. Le jardin nous fait prendre conscience des sentiments le plus réjouissants comme : d'amour (jardin d'amour) et des passions le plus effrayantes, t-elles de la mort (jardin de cimetière). Mais puisque la question du « jardin » devait s'en tenir aux expériences qui permettent de faire ressortir la pensée du « là » de milieu sédentaire de précarité qui l'angoisse et l'ennui et le pousser, envoyer vers l'inconnu, de le décentraliser; l'éclaircie d'être là, au milieu, qui est toujours dans l'homme, et avec qui se déplace, ne se trouve pas seulement dans lui. Il est important de reconnaître que le jardin est aussi un événement de vérité : « $\dot{\eta} \alpha \lambda \dot{\eta} \vartheta \varepsilon\llcorner\alpha\rangle$ sachant qu'elle provient de négation du Léthé, qui signifie en grec l'oubli. Il n'est pas seulement le produit d'une création géniale, héritée des dieux ou de dieu, mais aussi une œuvre qui possède en elle - même sa propre luminosité (delos). Il nous guérit par cette lumière comme Orphée guérit par la sienne, Orphée (du phénicien: «Aour» la lumière et « Rophae» guérison, celui qui guérit par la lumières). Le jardin qui nous entoure de partout, aux yeux des certains hellénistes disparaît au point de ne pas exister. Pour eux la contemplation d'une grandiose architecture grecque, des temples, des théâtre, des portiques, situés au milieu des contrées montagneuses, efface la modestie des jardins, en faisait d'eux les constructions trop fragiles, arrachées du monde hérö̈que grec. Le jardin, plus que les pierres assemblées en un mur de la cité, est porteur d'une renversante intensité des forces élémentaires de la terre et de ciel. Il est beaucoup plus présent, au sens où il accède à leurs essences véritables. Le jardin, c'est un événement qui ouvre et qui établit un monde, un événement pratiqué par l'homme, c'est-à-dire qu'il tient dans ses mains.

Le jardin a un lieu et un jour. On ne sépare jamais la coïncidence du jardin et d'exergue topographique. Phénoménologie du jardin, jardin comme phénoménologie, énigme du jardin dans la géographie du paraître. La lumière (phos, d'où : phainesthai, phantasme, qui veut dire spectre, la fantaisie) commence le discours philosophique, comme discours d'une révélation, d'une possibilité de manifestation. Nous avons cherché sa définition la plus originaire, c'est-à-dire plus proche de la source, de la seule et même source. Cette lumière du jardin nous dicte ce que hier encore, chez nos ancêtres Grecs, on croyait naïvement soustraire, voire éliminer. Alors il faut repenser le passé et l'avenir du jardin. N'oublions pas que nous ne disposons d'aucun terme commun pour désigner le jardin même, le langage indo-européen ne se rassemblait pas sur sa notion et l'assurance de 
penser l'enclos limité paraît largement excessive et plus que problématique. Tous les mots réunis pour parler de jardin, de $\chi \tilde{\eta} \pi 0 \varsigma$ à $\eta \vartheta o \varsigma$ par $\chi \omega ́ p \alpha$ nous invitent à designer un horizon. Dans cet horizon, dans cette lumière, nous avons trouvé notre lieu aporétique, sans issue, sans chemin assuré, sans route ni arrivée, sans dehors ni dedans. Notre jardin figure cet horizon. Mais n'oublions pas qu'il s'agit de penser et de dire, dans les limites assignées, une certaine absence d'horizon ; paradoxalement, l'absence d'horizon conditionne l'avenir même. Le surgissement de l'événement doit trouver tout horizon d'attente. La pensée philosophique doit trouver son jardin d'attente, pour une rencontre véritable. D'où appréhension d'un abîme en ces lieux, par exemple le jardin dans le jardin, la forêt dans la forêt et le désert dans le désert. Là où l'on ne peut ni doit voir venir ce qui devrait ou pourrait peut-être venir. Ce qui reste à laisser venir.

Philosopher c'est un art du voyage, du tour et du détour, qui nous approche et éloigne du jardin. Nous avançons vers le jardin, nous passons d'une étrangeté à une autre, nous enchaînons mystère après mystère comme un jardinier qui s'étonne de la croissance de ses plantes car comme l'enseignait Epicure : $\tau \tilde{\alpha} \varsigma$

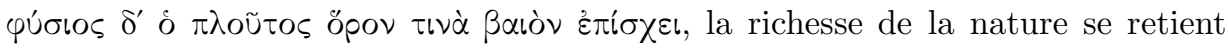
dans un petit enclos. ${ }^{25}$ Ce jardinier nous invite à un exercice difficile. Il nous faut devenir attentifs à ce qui ne se montre que dans le silence et y surprendre cependant l'exigence d'une parole qui l'exprime. Pour rendre cette difficulté sensible, nous avons pris comme exemple ce qui est maintenant immédiatement à notre portée - le jardin. Le mot et avec ce mot la chose. Il ne s'agit plus des écritures, mais du bloc massif des interprétations des discours, des thèmes, des modes de représentation, produits et entassés à propos de ce que nous nommons le « jardin ». Nous sommes entre jardin réel et faux-semblant, et parfois nous ne savons pas les distinguer, puisque le « réel d'en haut» dévoile le « réel d'en bas » comme illusoire et vice versa. Nous nous mettons débout, et regardons au-dessus des plantes, comme les gens de la caverne platonicienne, nous tournons notre regard vers la vérité qui éblouit. Pour observer à partir de la Lune : la Terre, le Monde, le Jardin il faut se procurer un télescope ${ }^{26}$, mais pour voir du jardin

25 Diogène Laërce, La vie des hommes illustres, traduction de Robert Genaille, 1933.

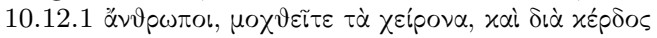

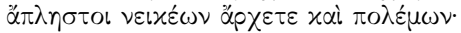

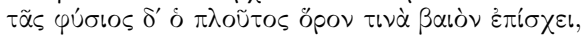

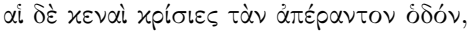

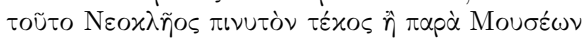

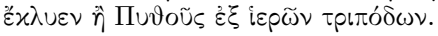

26 Voir le progrès scientifique, comme processus décrit par Hannah Arendt, en particulier l'invention du télescope qui a eu pour effet de permettre à l'homme d'avoir un point d'Archimède pour observer la Terre de l'extérieur. Pour Arendt les développements de la philosophie ne sont que le prolongement de cette aliénation du monde. 
Le Monde, La Terre et La Lune, il suffit de tourner son regard. Le philosophe n'a plus besoin de se détourner du monde périssable des illusions pour pénétrer dans le monde des vérités éternelles, il se retire dans le jardin où la pensée est perçue comme une manière d'atteindre l'éternité.

Deleuze dit que nous sommes des nomades. Et le jardin nomade n'est pas celui à porter avec nous, ni à trouver à l'endroit de déplacement. Restons fidèles à notre nature vagabonde, pour qu'elle « ici », « ailleurs », « maintenant », «plus tard», n'ait pas un sens absolu et fixe. Certains qui se croient installés ne possèdent pas de jardin. Ils jouissent de leur installation, qui leur donne une arrogance peu supportable aux autres, qu'ils excluent de leur lieu fermé, de leur jardin fermé, dans le moment même où ils feignent de les y admettre. Tel est le cas des philosophes sédentaires. Ils n'avaient d'autre but que de nous persuader de leur gloire, de leur certitude. Sans doute pensaient -ils que nous en garderions pour toujours la nostalgie et que, revenus du voyage, nous raconterions l'histoire de leurs merveilles un moment partagées, ruminant sans cesse en nous-mêmes l'amertume d'en avoir été séparés. Nous sommes comme des «sceptiques, une variété de nomades qui détestent s'établir durablement quelque part, rompirent périodiquement l'association civile. ${ }^{27}$ Nous détestons vivre dans le positif et absorber les événements et les choses comme si le cours du monde nous les devait, nous ne sommes pas persuadés que le monde même nous a élus pour une telle jouissance.

Nous autres, vagabonds de nature, nous ne pouvons vivre que dans la négation. Dans la mesure, ou nous ne sommes pas nombreux, nous ne pouvons pas empêcher « les dogmatiques d'essayer de restaurer - l'association civile, ${ }^{28}$ comme celle d'agora, «toujours de nouveau, sans disposer pour autant d'un plan sur lequel ils se fussent accordés. ${ }^{29}$ Nous sommes en état de transfert. 《Ici », 《 ailleurs », « maintenant », « plus tard , ces lots de notre langues désignent pour nous des états «métastables» qui passent sans cesse les uns dans les autres : ici est tout autant un ailleurs que maintenant un plus tard. Nous n'avons pas de résidence assignée ni de jardin clos. Il n'y a pas de lieu qui nous attend, lieu immobile, ni dans la ville visible ni dans celle invisible, il n'y a que « au-delà » du jardin de rencontre philosophique, entrevu de ce point où nous nous tenons maintenant. Pour les gens de passage, tout jardin est effet de perspective, qui sans cesse modifie l'horizon. Nous ne l'oublions pas en parlant de mirages et n'en croyons pas nos yeux. Sans doute avions-nous trop accordé de confiance aux gens de

27 Emanuel Kant, Critique de la raison pure, Traduction et présentation par Alain Renaut, Flamarion, 2006, p. 64.

28 Ibidem, p. 64.

29 Ibidem, p. 64. 
l'agora, écrasés que nous étions sous l'abondance permanente de leurs signes, qui ne renvoient qu'à eux-mêmes et se ferment sur leur propre compacité. Un temps nous avons vécu médusés et emprisonnés. Oublions la ville et sortons au-delà de l'agora! Soyons les voyageurs du Gai Savoir ${ }^{30}$, ceux qui cherchent à se rendre compte de la hauteur des tours d'une cité, la quittent. Où voyageons nous ? Justement dans le champ de nos propres jardins, dans les paroles et les écritures, comme si chaque phrase dite forait devant elle un creux, d'où jaillirait un autre jardin, une autre terre fertile. Et plus nous nous éloignonsde jardin, plus le jardin viendra avec nous. Nous sommes obligés de penser ce rapport du jardin. Le jardinage nous donne à penser. Le jardin nous enveloppe et il est aussi le chemin. Il est une route qui ferme, qui encercle mais qui aussi ouvre. Il faut alors prendre pied et s'y trouver et en même temps il faut y prendre son envol, et s'en aller. A la fois s'en aller et rester. Le jardin nous enveloppe, c'est notre jardin, notre jardinage. Nous ne pouvons pas en sortir, il nous accompagne partout. Nous sommes tous jardiniers au sens propre. Nous sommes obligés de cultiver nos plantes sur notre peau, de cueillir et de lire sur la peau des autres, leurs mots, leurs sentiments, leurs plantes. Nous sommes toujours dans ce rapport à la fois d'exclusion et d'intériorité du jardin. L'intérieur et l'extérieur se tiennent ensemble. La notion de frontière du jardin doit être pensée entièrement, elle n'est pas une ligne de séparation, mais une relation mobile, un horizon mobile. A l'horizon les plantes se dessinent toujours dans la lumière du jour. C'est le désir de la forme qui échappe au crépuscule pour se manifester de nouveau à l'aube dans le jardin. Quand les plantes, les choses se dessinent, cela veut dire qu'elles se dévoilent. C'est la lumière du soleil qui leur donne la vie, la vérité qui est décisive du point de vue du désir de philosophie.

\section{CONCLUSION: PHILOSOPHIE DE JARDIN}

La réalité du jardin que je propose est déjà un résultat qui n'apparaît que par une perspective, une structure. La pensée de jardin est donc une pensée de la réalité comme interprétation, tout ceci supposant que toute interprétation n'existe qu'en tant que perspective. À partir de cette thèse perspectiviste, la question qui se pose (comme elle s'était posée à Protagoras, cf. le dialogue de Platon) est de savoir si toutes les perspectives (ou interprétations) de jardin se valent.

Jardin déconstruit, en tant qu'objet d'abord énigmatique malgré les références immédiates à Epicure, l'espace articulé par l'architecture, la sculpture, la

30 Voir l'aphorisme de F. Nietzsche du Gai Savoir nr. 380. 
peinture et la poésie qu'un auteur comme Hegel avait relu comme structurant une histoire de l'esprit dans le monde grec. Il conduit à définir, depuis la difficulté même d'identifier ce qu'il nomme dans cet espace et qui ne semble correspondre à rien de ce que nous nommons «jardin» dans notre propre structuration des lieux du vivre et du penser, des situation originales et non encore découvertes de ce que la Grèce antique expérimentait à l'aurore de nos propres gestes.

Le «jardin» permet à nouveau et autrement qu'en référence à l'agora d'effectuer la reprise d'élan dans les commencements grecs de la pensée que menèrent avec des enjeux différents Nietzsche ou Heidegger. Il permet de revenir sur les spatialités au fondement des remobilisations de l'espace public antique mises en œuvre par Hannah Arendt. Le «jardin» interroge plus profondément. Comme disait Foucault «Le jardin, c'est un tapis où le monde tout entier vient accomplir sa perfection symbolique, et le tapis, c'est une sorte de jardin mobile à travers l'espace. Le jardin, c'est la plus petite parcelle du monde et puis c'est la totalité du monde. ${ }^{31}$

L'espace ouvert par la politique est un espace de parole partagée : il n'existe que par la discussion publique entre les citoyens, qui transforme les opinions subjectives en éléments d'une réflexion objective sur le bien commun. Les Grecs ont inventé la cité, la polis pour préserver les conditions politiques d'existence. La polis avec l'agora au milieu est le premier espace du politique. Deleuze écrit : „Si la philosophie a une origine grecque autant qu'on veut bien le dire, c'est parce que la cité, à la différence des empires ou des États, invente l'Agôn comme règle d'une société des amis, la communauté des hommes libres en tant que rivaux (citoyens)." 32 C'est dans le cadre d'un agon (concour, réunion) que sont representé tragédies, drames satyrique, comédies. Son modèle se retrouve déjà dans l'Iliade et l'Odyssée. C'est au cours d'agon que se déroule les épreuves des jeux organisés lors des funérailles de Patrocle ${ }^{33}$ et d'Achille ${ }^{34}$ Mais entre l'assemblée des guerriers, l'assemblée des citoyens dans l'Etat oligarchique et l'Ekklesia démocratique qui s'assemble sur l'agora, il y a une continuité évidente. Car le débat politique constitue une lutte codifiée qui rapelle celle mise en oeuvre lors des jeux funéraires, et dont sont les avatars, les différents concours organisés par la cité. La cité de la polis grecque est néanmoin fondée sur la persuasion et non la violence, état de risque se tiens au dehors de la polis et au dehors du jardin encore que le mot

\footnotetext{
31 Michel Foucault, Dits et écrits 1984, Des espaces autres (conférence au Cercle d'études architecturales, 14 mars 1967), in Architecture, Mouvement, Continuité, n ${ }^{\circ}$ 5, octobre1984, pp. 46-49.

32 G. Deleuze, Les conditions de la philosophie, Qu'est-ce que ma philosophie ? Texte publié initialement in Chimères, n 8, mai 1990.

33 Homère, Iliade, XXIII 507, 685, 710, 799.

34 Homère, Odyssée, XXIV, 80-89.
} 
"jardin" soit trompeur car ces "terres" apparemment immobiles ne cessent d'être bougées pour donner l'expression de la capacité d'agir (agôn) de la nature. L'art dialectique qui se manifeste à travers le combat agonistique produit la réthorique qui est encore agonistique mais plus indirecte. La réthorique, c'est la dialectique plus élément émotionel qui donne la persuasion. Epicure dit qu'il faut aussi persuader la nature pour produire le jardin. C'est la vie harmonisée avec la nature de la vie du jardin qui fructifie un nouveau ethos. Pour le saisir il suffit de sensibiliser l'atmosphère du jardin. Il faut persuader la nature. « Il ne faut pas faire violence à la nature, mais la persuader : nous la persuaderons en contentant les désirs nécessaires, et aussi les désirs naturels s'ils ne sont pas nuisibles, mais en repoussant durement les nuisibles. ${ }^{35}$ Epicure domestique la nature pour aboutir au « jardin ».

L'invention des Grecs a été de conserver la mémoire de mise en milieu du sceptre de l'archè. Le pouvoir n'appartient à personne, il est au milieu. L'homme n'est pas un être politique naturellement, puisque c'est l'agora, la politique qui le définit comme création d'un monde humain en dehors de la nature. Sorti du politique, l'homme retourne en quelque sorte à l'animalité, mais en passage il s'arrête dans le jardin où il constitue sa nature humaine. La création d'un espace politique, n'est pas un seul lieu véritable d'expression de la qualité humaine de l'homme, en ce qu'il rend possible la fondation d'un monde commun régi par le nomos. A côte de l'agora, là où les hommes se reconnaissent mutuellement comme des citoyens, se trouve le jardin où les hommes sont ensemble sous l'horizon d'un monde commun - d' « être-ensemble ».

Rapellons que dans «Les Lois», le dernier dialogue de Platon, qui touche au problème de la meilleure constitution politique, trois personnages, un Athénien (Socrate n'apparaît pas de nom) un Crétois nommé Clinias et un Lacédémonien nommé Mégillos, partent de Cnossos, la ville de Minos, pour aller visiter dons la montagne Ida le jardin de Zeus. Cet endroit mythique fait référence au mariage paradigmatique de Zeus et d'Héra. Ce jardin de mariage sacret et le temple de Zeus se présentent comme l'objectif à atteindre. Les amis sortent de la ville et se dirigent vers le jardin, pour atteindre la vérité.

Ce travail encourage à un déplacement en direction du dehors. La cité grecque est remplacée par des citadelles intérieures, des exercices spirituels, des sectes philosophiques, des prédications errantes. La recherche du jardin grec s'inscrit dans l'étude de ce déplacement. Se saisissant de la question du jardin, elle se tient d'emblée hors des catégories les plus immédiatement à même d'accueillir le thème

35 Epicure, Sentences Vaticanes. 21, trad. Marcel Conche, édit et trad. de Epicure, Lettres et

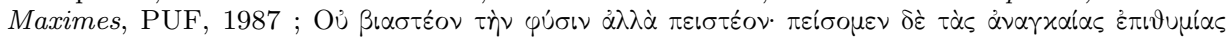

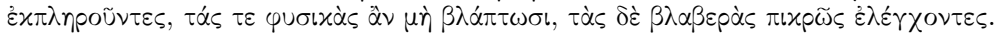


de la soutenance de la centralité opérée par la réévaluation du motif de l'agora telles que l'intériorisation spirituelle du lieu de la pensée ou ses extériorisations sociales et géographiques sur les chemins ouverts du monde hellénistique.

Le jardin, envisagé dans la conception courante: fermé, nous apparaît comme ouvert chez les Grecs, parce que ses limites ne jouaient pas le même rôle qu'aujourd'hui. Le jardin grec n'est pas le jardin d'histoire, tel connu de la tradition hebraique, parceque les limites du jardin grec sont davantage la réfutation d'un enclos, elles nient sa fonction qui limite le champs visuel. Nous voulons dire à ce moment qu'un philosophe grec « enfermé » dans le jardin regarde là où la vue n'arrive plus à voir. Il titube au dehors de la caverne platonicienne, parce qu'il n'est pas du tout enchaîné au mur, dans son jardin il n'y a pas de mur. On ne peut penser que sur cette ligne ; penser, c'est voyager, en s'approchant du voyageur nietzschéen qui poursuit son ombre. Voyager en étant immobile, sans franchir la ligne frontale de la vie.

Étant donné que le jardin n'est pas un objet dont la philosophie constituerait le sens ou la vérité, il est un domaine de pensée autonome et indépendant. La philosophie se sert du jardin, de son espace et de ses œuvres, du contact avec ceux-ci afin de produire des effets, des pensées, des concepts ou des idées. En fait, le rôle que nous pouvons aujourd'hui accorder à la philosophie du jardin, c'est celui d'accompagner le développement d'arguments et de raisons à l'égard du projet durable. La philosophie du jardin doit, en ce sens, nous aider à ouvrir et universaliser nos convictions, nos valeurs et nos conceptions préétablies. Précisons que la philosophie du jardin doit servir à identifier et à reconnaître les différents paramètres culturels, naturels ou philosophiques qui œuvrent dans ce domaine. En fait, la philosophie du jardin doit nous permettre de nous accoutumer avec les différentes façons qu'une personne peut avoir de concevoir la relation entre philosophie et nature, morale, société et individu, ou encore d'autres topos du même type. De cette façon, la philosophie du jardin ne travaille pas sur les qualités formelles du jardin, laissant celles-ci volontairement aux « sciences » de l'horticulture, mais accompagne la réflexion relative aux sens et aux horizons du projet horticole moderne.

Cette fondation d'un lieu spatial, temporel et solitaire rend possible l'ouverture à autrui, la rencontre avec autrui, la rencontre philosophique, non nécessairement à penser en termes de conflictualité irréductible, mais davantage en harmonie dans un ensemble de différences de tonalité. Pour conclure je citerai un philosophe polonais Kolakowski qui dans une posture ironique se moque de jardin : «Malgré de multiples formules on a échoué à créer une définition satisfaisante du jardin et du jardinage. Toutes ces définitions laissent un territoire étendu d'incertitude par rapport à la question du jardin ; quels sont les phénomènes qui appartiennent au champ d'investigation. Tout simplement, nous 
ne savons pas précisément ce que sont le jardin et le jardinage. L'utilisation de ces notions est intellectuellement risquée comme le sera d'autant plus la cultivation réelle du jardin. Tu ne vas pas cultiver le jardin ! QUOD ERAT DEMONSTRANDUM $»$.

\section{Summary}

All philosophical problems appear in a specific situation and establish our relations to social and natural environment. Greek philosophy is usually associated to Agora, the place where the people gather together to exchange their views. I try to put it in a different context - out of the city and its institutions, and I tend to abandon the idea of polis as a "universal pattern of being and thinking". Yet to conceive properly Hellenistic philosophy we have focus on garden situated between bios et zoe, extra muros, where: $\tau \tilde{\alpha} \varsigma$

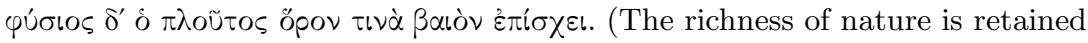
in a small enclosure.) The idea of delimitation of garden has to be rethought, considering its ontological and epistemological mobility, which refers to the notions of... (revolutionary garden, Dasein, stay, lignes of escapes, hétérotopie, khôra, not cultivated garden) - the milestones of postmodern philosophical reflection.

\section{Bibliographie}

Theodor W. Adorno et Max Horkheimer, La dialectique de la raison, Gallimard, Paris, 2004.

Hannah Arendt, Condition de l'homme moderne, traduction française G. Fradier, Calmann-Lévy, 1961, 1983, réédité avec une préface de Paul Ricoeur, Pocket, 1988, 1992.

Anatole Bailly, Dictionnaire grec français, Librrairie Hachette, 1957.

Helmutt Baumann, Le Bouquet d'Athéna les plantes dans la Mythologie et art grecs, Maison Rustique, 2008.

Luc Brisson, Platon, Oeuvres complètes, Flammarion, 2008.

Marcel Conche, édit et trad. de Epicure, Lettres et Maximes, PUF, 1987.

Gilles Deleuze, Dialogues, avec Claire Parnet, Ed. Flammarion, Paris, 1977.

Gilles Deleuze, Différences et répétitions, Presses Universitaires de France, Paris, 1968.

Gilles Deleuze, F. Guattari, Félix, Capitalisme et schizophrénie. L'Anti - Edipe, Ed. Minuit, 1972. 
Gilles Deleuze, Dialogues, avec Claire Parnet, Ed. Flammarion, 1977.

Gilles Deleuze, Les conditions de la philosophie, Qu'est-ce que ma philosophie ? Texte publié initialement in Chimères, no 8, mai 1990.

Jean-Toussaint Desanti, Réflexion sur le temps, Conversation avec Dominique-Antoine Grisoni, Grasset, 1992.

Michel Foucault, Dits et écrits 1984, Des espaces autres (conférence au Cercle d'études architecturales, 14 mars 1967), in Architecture, Mouvement, Continuité, no 5, octobre 1984.

Marguarite Garrido - Hory, Antonio Gonzalès, Histoire, espaces et marges de l'antiquité : hommages à Monique Clavel-Lévêque, volume 4.

Pierre Hadot, Exercices spirituels et la philosophie antique, Albin Michel, 2002.

Martin Heidegger, Séjour, Aufenthalte, Edition bilingue, traduction, postface et notes de François Vezin, Editions du Rocher, Paris, 1992,

Homère, Odyssée, trad. Philippe Jaccottet, édit La Decouverte, Paris, 1992.

Homère, Iliade, trad. Paul Mazon, Gallimard, 1975.

Homeri, Odyssea, éd. von der Mühll, P., Helbing \& Lichtenhahn, Basel, 1962.

Emanuel Kant, Critique de la raison pure, Traduction et présentation par Alain Renaut, Flamarion, 2006.

Diogène Laërce, La vie des hommes illustres, traduction de Robert Genaille, 1933.

Friedrich Nietzsche, Gai Savoir, Nouvelle édition des œuvres philosophiques complètes de Nietzsche. Traduction française des textes établis par G. Colli et M. Montinari. Paris, Gallimard: Gallimard, 1967-1997.

Pindar, Odes - Les Pythiques,

Platonis opera, éd. Burnet, J. Oxford: Clarendon Press, 1901, Repr. 1967.

Pline L'Ancien, Histoire Naturelle, En collaboration avec Agnès Vinas, (site Méditerranées), traduction, E. Littré,

Pline l'Ancien, Histoire Naturelle, éd. d'Émile Littré.

Plotin, Ennéades, trad. Jérôme Laurent, Sur lebeau, dans Plotin, Sur le beau et autres traités, trad. sur la dir de Luc Brisson et Jean-François Pradeau, Flammarion, 2008.

Plotinus, Enneades, Ed. Henry, P., Schwyzer, H.-R. Leiden: Brill, 1:1951; Ennead $1,6,7,17$.

Jean Pierre Vernant, Mythe et religion en Grèce ancienne, édition du Seuil, 1987.

Obraz parku - ogrodu a alternatywa ewolucji literackiej, w: Przestrzen ogrodu, przestrzen kultury, Universitas, Kraków, 2008.

Textes grecs selon TLG, the Packard Humanities Institute, The Perseus Project and others. Dans Diogenes version 3.1.6. 